there are a number of commercial assays reporting various mutations linked to fluoroquinolone resistance. This talk will look at the evidence supporting the use of these assays and how these assays may contribute to patient management.

\section{S03.3 MYCOPLASMA GENITALIUM: NEW TREATMENT APPROACHES}

C Bradshaw*. Alfred Health and Monash University, Melbourne, Australia

10.1136/sextrans-2021-sti.28

Mycoplasma genitalium is an increasingly prominent STI notable for its limited susceptibility to antimicrobials and increasing acquisition of antimicrobial resistance. While AMR varies globally, rising rates significantly impacts on the efficacy of available antimicrobials and clinicians now commonly infections that challenging to treat. This talk will discuss the range of current and new treatment strategies including resistanceguided treatment strategies, combination treatment approaches, repurposed antimicrobials and new agents

\section{S03.4 M. GENITALIUM: IS IT TIME FOR SURVEILLANCE?}

L Manhart*. University of Washington, Seattle, Wa, USA

10.1136/sextrans-2021-sti.29

First identified in 1980, Mycoplasma genitalium is a recognized cause of male urethritis and is associated with increased risk of female upper reproductive tract disease and sequelae. Coinciding with the recognition of these disease associations is the emergence of significant antimicrobial resistance and potentially untreatable infections. Globally, from 2016-2017, half $(51.4 \%)$ of $\mathrm{M}$. genitalium infections were resistant to macrolides, $\sim 9 \%$ contained fluoroquinolone resistance-associated mutations, and markers of dual class resistance were detected in $\sim 4 \%$ (Machalek, 2020). Yet these estimates are derived largely from dedicated research studies, which are not always able to recruit and enroll representative samples of the population at risk. Surveillance systems overcome some of these limitations and are beginning to be implemented in several countries. In the US, a pilot sentinel surveillance system to monitor the prevalence of infection, and track changes in antimicrobial resistance was launched in 2020. Remnant specimens from patients tested for Neisseria gonorrhoeae and/or Chlamydia trachomatis attending eight sexual health clinics in four geographic regions of the US are tested for M. genitalium using the Aptima Mycoplasma genitalium assay (Hologic, Inc.). Each clinic submits 400 specimens: 200 from male sex at birth patients; 200 from female sex at birth patients (100 symptomatic and 100 asymptomatic for each sex) for a total of 1600 specimens per year. Macrolide and fluoroquinolone antibiotic resistance markers at positions 2058 and 2059 in M. genitalium 23S rRNA and in parC mRNA, respectively, will be assessed. The prevalence of $\mathrm{M}$. genitalium infections, prevalence of antibiotic resistance, and characteristics associated with resistant infections from the 2020 surveillance cycle will be presented. These results will be discussed in the context of surveillance efforts in other settings.
S04.2 HOW SHOULD ANTIMICROBIAL RESISTANCE IN NEISSERIA GONORRHOEAE INFLUENCE PID TREATMENT

J Ross*. University Hospital Birmingham NHS Trust, Birmingham, UK

10.1136/sextrans-2021-sti.30

Rising levels of antibiotic resistance in uncomplicated $\mathrm{N}$ gonorrhoeae infection have led to changes in recommended treatment and suggestions for new management paradigms. It is, however, unclear how these new approaches to treatment should be reflected in managing gonococcal pelvic inflammatory disease (PID). Gonorrhoea remains an important but relatively uncommon cause of PID for which treatment is often commenced prior to identification of the causative organisms. Those at highest risk (severe symptoms, partner with gonorrhoea, gram negative diplococci on microscopy) should have therapy which includes cover for $\mathrm{N}$ gonorrhoeae. Newer agents, such as lefamulin, gepotidacin and zoliflodacin have not been evaluated in women with pelvic infections but frequently have a wide spectrum of activity covering not just gonorrhoea but many of the other bacteria associated with PID, and achieve good tissue levels in the upper genital tract. However, optimal dosing regimens for PID to achieve clearance of infection but minimise the risk of developing future resistance need to be determined.

\section{S04.4 EVIDENCE BASED APPROACHES TO PREVENTING PELVIC INFLAMMATORY DISEASE (PID) AND ITS SEQUELAE}

P Oakeshott*. St George's University of London, London, UK

\subsection{6/sextrans-2021-sti.31}

There are many challenges to preventing pelvic inflammatory disease (PID). The case definition is unclear: clinically diagnosed PID may not be confirmed by laparoscopy/endometrial biopsy/imaging. Both PID and its main cause Chlamydia trachomatis infection can be asymptomatic, and often no clear pathogen is identified. Most women (>85\%) with chlamydia infection do not get PID. Finally, uptake of chlamydia screening is often low, and treatment of those infected and their sexual partners can be challenging.

A 2016 Cochrane review found four trials (in 21, 686 women) which examined the effect of chlamydia screening on PID: Scholes, Ostergaard, Oakeshott, Andersen. Overall, the risk of PID was lower in women in intervention than control groups: RR $0.68,0.49$ to 0.94 . But in the two trials at low risk of detection bias (Oakeshott, Andersen) the RR was $0.8,0.55$ to 1.17 , compatible with no effect. Thus, although detection and treatment of chlamydia infection can reduce the risk of PID in an individual woman, the size of the effect is uncertain. It is also unclear whether chlamydia screening to prevent PID reduces rates of infertility and ectopic pregnancy.

Our recent 'Test $\mathrm{n}$ Treat' trial of free rapid chlamydia tests and same day treatment in 500 sexually active, ethnically diverse teenagers, highlights the problems of persuading those at risk to get screened. Despite a $6.2 \%$ prevalence of chlamydia (and $0.6 \%$ gonorrhoea), test uptake was only $13 \%$. Interviews suggested this was due to not feeling at risk, perceptions of stigma and little knowledge of STIs. However, $£ 5$ notes can be effective incentives, and enabled us to recruit 